Please do not remove this page

RMIT

UNIVERSITY

\title{
A gradient-based weighted averaging method for estimation of fingerprint orientation fields
}

Wang, Yi; Hu, Jiankun; Schroder, Heiko

https://researchrepository.rmit.edu.au/esploro/outputs/9921862100801341/filesAndLinks?institution=61RMIT_INST\&index=null

Wang, Y., Hu, J., \& Schroder, H. (2005). A gradient-based weighted averaging method for estimation of fingerprint orientation fields. Proceedings of the Digital Imaging Computing: Techniques and Applications, 20-29. https://doi.org/10.1109/DICTA.2005.4

Published Version: https://doi.org/10.1109/DICTA.2005.4

Repository homepage: https://researchrepository.rmit.edu.au

(c) 2005 IEEE. Personal use of this material is permitted. However, permission to reprint/republish this material for advertising or promotional purposes or for creating new collective works for resale or redistribution to servers or lists, or to reuse any copyrighted component of this work in other works must be obtained from the IEEE.

Downloaded On 2023/04/26 22:25:13 +1000 


\title{
A Gradient Based Weighted Averaging Method for Estimation of Fingerprint Orientation Fields
}

\author{
Yi Wang, Jiankun Hu, Heiko Schroder \\ School of Computer Science and Information Technology \\ Royal Melbourne Institute of Technology \\ Melbourne VIC 3000, Australia \\ \{alice, jiankun, heiko\}@cs.rmit.edu.au
}

\begin{abstract}
Estimation of orientation fields is an essential module in a fingerprint recognition system. Conventional gradient based approaches are popular but very sensitive to noise. In this paper, we propose a new implementation that is more resistant to noise. Our basic idea is to conduct redundant estimation over four overlapping neighborhoods for each target block. Following this idea, we devise a weighted averaging scheme operated on the base blocks directly. Thus, each block (including the target one) in the overlapping neighborhoods has different impact on estimation of the dominant orientation fields. Our preliminary experiment results suggest that the proposed weighted averaging algorithm is more robust against noise in comparison with other gradient based methods.
\end{abstract}

\section{Introduction}

Fingerprints are constructed by ridges and furrows. They are permanent physiological features of human beings. It is well established in science that ridge structures are highly distinctive between individuals [10]. This has enabled fingerprints to be a reliable means for personal identifications. Today, automated fingerprint identification systems (AFIS) have been widely used in forensic science and airport access control.

Fingerprints are usually acquired by impressing fingertips on sensor platens of acquisition devices. The quality of fingerprint images depend on acquisition environments, skin conditions and user interactions. Dirt, moist, creases and cuts on the surface of fingertips produce noise in fingerprint images. The noise effects can cause uncertainties in feature extraction of fingerprints, thus increase the error rate of the identification system.

This is especially true for the emerging applications in

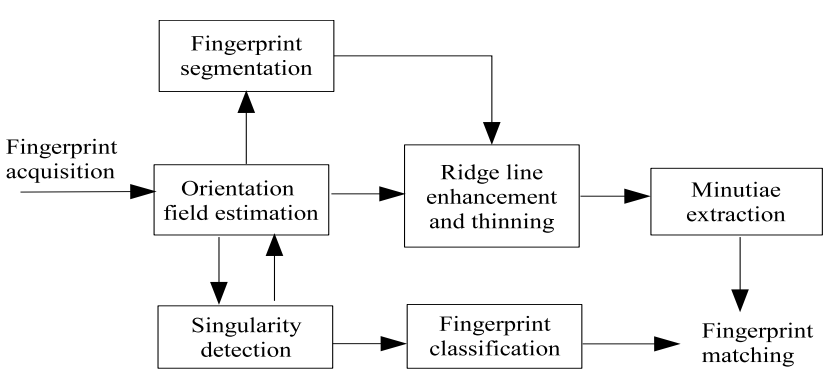

Figure 1. Flowchart of a general fingerprint recognition system.

embedded security, where the acquisition environments are not as well controlled as those of the current AFIS systems. In the embedded applications, fingertips and sensor platens are less likely to provide good quality fingerprint inputs as in the use by official organizations, such as police office and customs. It means more noise can be introduced during fingerprint acquisition. In this paper, we consider noisy fingerprint inputs for fingerprint recognition.

Fingerprint recognition refers to a series of processes of extracting the traits in a fingerprint image and presenting them in quantitative forms. It may also include identifying the classes of ridge patterns, which is useful in discriminating fingerprints. A simple flowchart of general fingerprint recognition systems is displayed in Figure 1.

As shown in Figure 1, the estimation of orientation fields is an essential module in a fingerprint recognition system. The orientation fields represent the ridge flow directions on regularly spaced grids. They help to reveal intrinsic features of ridge topologies, and thus have great impact on almost all subsequent processes. For example, it helps to enhance the clarity of ridge structures $[3,12]$ and is used to detect fingerprint singularities $[1,7]$.

To estimate fingerprint orientation fields reliably, the 
most popular approach is to use gradient based methods $[1,4,6,11]$. Although there are other approaches proposed in the literature $[5,9,13,15]$, they do not provide as much accurate results as the gradient based methods because most of them rely on a fixed number of filters and templates [8]. Some of the methods are also known to be computationally expensive as they involve exhaustive comparing processes [16].

The gradient based methods also have their drawbacks. Since the gradients of gray intensity are usually computed on pixel level, the gradient based methods are sensitive to noise. Therefore, it often entails post processing techniques in addition to the gradient based methods, especially when the quality of a fingerprint image is very poor. In such cases, the gradient based algorithms often serve as coarse orientation field estimators to detect fingerprint singularities and to feed the post processing module with initial statistics. Hence, a more accurate estimation of coarse orientation fields can increase the accuracy and reduce the computation burden of the post processing stage.

In this paper, we propose an enhanced gradient based method for estimation of fingerprint orientation fields. Our basic idea is to conduct redundant estimation over four overlapping neighborhoods for each target block. Following this idea, we devise a weighted averaging scheme that can be operated directly on the base blocks.

The rest of this paper is organized as follows. Section 2 introduces the technical background and related work in the literature. Section 3 presents our weighted averaging method. Experiment results are shown in Section 4. Finally, we summarize our work in section 5 .

\section{Related Work}

In gradient based methods, the gradient vectors are first calculated for a fingerprint image by taking the partial derivatives of gray intensity at each pixel. A gradient vector can be denoted as $\left[g_{x}, g_{y}\right]^{T}$ in Cartesian coordinates. In a fingerprint image, the gradient vectors always point to the directions of the highest variation of gray intensity, which are perpendicular to the edges of ridge lines.

A fingerprint orientation map is defined as a collection of two-dimensional orientation fields. The magnitudes of these fields can be omitted. Only the angle information is of interest because it captures the dominant ridge direction in every regular spaced grid. An orientation map is commonly represented in the form of a matrix $\left\{\theta_{x y}\right\}$, where $\theta_{x y} \in[0, \pi]$. Denote the averaged gradient angle computed from the local gradient vectors in a grid as $\bar{\varphi}$. The dominant orientation angle $\theta$ is then orthogonal to $\bar{\varphi}$.

Since a ridge line has two edges, the gradient vectors at both sides of a ridge are opposite to each other. If we calculate $\bar{\varphi}$ by averaging the gradient angles directly, the opposite gradients at both sides of the ridge line are likely to cancel each other. To solve this problem, Kass et. al [6] proposed a simple yet effective idea of doubling the gradient angles before averaging. In this way, $\varphi$ becomes $2 \varphi$ and $(\varphi+\pi)$ becomes $(2 \varphi+2 \pi)$ which is also equal to $2 \varphi$. In practice, $2 \varphi$ is the angle of a squared gradient vector $\left[g_{s x}, g_{s y}\right]^{T}$ that has the following relation with $\left[g_{x}, g_{y}\right]^{T}$ according to the trigonometric identities:

$\left[\begin{array}{l}g_{s x} \\ g_{s y}\end{array}\right]=\left[\begin{array}{l}g^{2} \cos 2 \varphi \\ g^{2} \sin 2 \varphi\end{array}\right]=\left[\begin{array}{c}g^{2}\left(\cos ^{2} \varphi-\sin ^{2} \varphi\right) \\ g^{2}(2 \sin \varphi \cos \varphi)\end{array}\right]=\left[\begin{array}{c}g_{x}^{2}-g_{y}^{2} \\ 2 g_{x} g_{y}\end{array}\right]$.

The averaged square gradients $\left[\bar{g}_{s x}, \bar{g}_{s y}\right]^{T}$ in a block $B$ can be therefore calculated by:

$$
\left[\begin{array}{l}
\bar{g}_{s x} \\
\bar{g}_{s y}
\end{array}\right]=\left[\begin{array}{c}
\sum_{B} g_{s x} \\
\sum_{B} g_{s y}
\end{array}\right]=\left[\begin{array}{c}
\sum_{B}\left(g_{x}^{2}-g_{y}^{2}\right) \\
\sum_{B} 2 g_{x} g_{y}
\end{array}\right] .
$$

Conventional gradient based methods $[1,6,11]$ divide the fingerprint image into equal-sized blocks comprised of $N \times N$ pixels, and perform the estimation of orientation field over each block independently.

In order to measure the reliability of estimation, Kass et. al [6] introduced a metric called coherence. The coherence metric calculates the strength of the averaged gradient in the distribution of local gradient vectors. For the same block $B$, it is given by [6]:

$$
\operatorname{Coh}_{B}=\frac{\left|\sum_{i=1}^{N} \sum_{j=1}^{N}\left(g_{s x}(i, j), g_{s y}(i, j)\right)\right|}{\sum_{i=1}^{N} \sum_{i=1}^{N}\left|g_{s x}(i, j), g_{s y}(i, j)\right|}
$$

Instead of operating with equal-sized blocks, Jain et. al [4] proposed a hierarchical scheme to dynamically adjust the estimation window via iterative steps. They introduced a concept of consistency, which is the normalized deviation between orientation estimates of the other blocks in a neighborhood and those of the centered target block. If the consistency level is above a predefined threshold value, the orientations around the target block are re-estimated in a smaller window size.

\section{The Weighted Averaging Method}

In general, the ridge flows are slowly varied across a fingerprint image except at several singular points. Therefore, fingerprints can be viewed as some oriented textures [11]. These textures usually exhibit local parallelism and have strong selectivity to orientations. In other words, the variation of gray intensities along a ridge flow is much slower than that in the perpendicular direction. This feature is termed anisotropy in texture analysis [6].

In this section, we propose an enhanced gradient based algorithm using a weighted averaging scheme that can exploit the above salient features of fingerprint ridge patterns. The algorithm is presented as follows. 


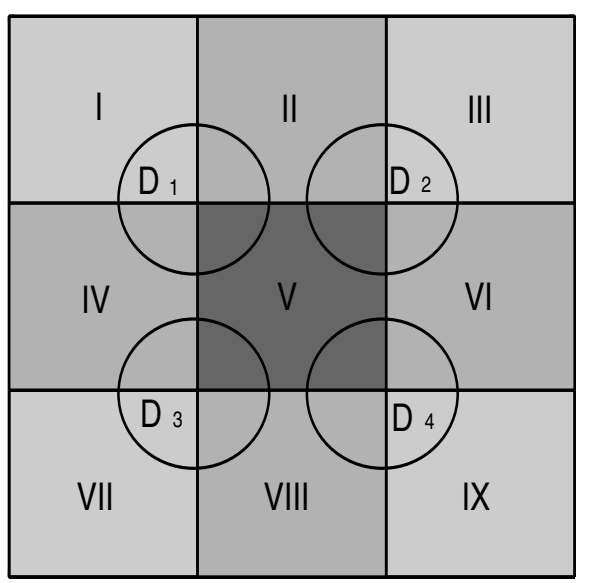

Figure 2. A site consists of $3 \times 3$ blocks with the target block $V$ in the center and four overlapping neighborhoods $D_{1}, D_{2}, D_{3}, D_{4}$.

We first divide the input fingerprint image into blocks each of size $N \times N$ pixels. We then group every $2 \times 2$ adjoining blocks into a neighborhood $D$ and every $3 \times 3$ adjoining blocks into a site with four neighborhoods overlapping on each other. An example of such organization is illustrated in Figure 2.

Consider the centered block $V$ in Figure 2 for which the dominant orientation field is to be estimated. Let blocks $\{I, I I, I V, V\}$ form a neighborhood marked $D_{1}$. Similarly, blocks $\{I I, I I I, V, V I\}$ form $D_{2}$, blocks $\{I V, V, V I I, V I I I\}$ form $D_{3}$, and blocks $\{V, V I, V I I I, I X\}$ form $D_{4}$. Note that the target block $V$ is included in all four neighborhoods but positioned at different corners. Using (1), the averaged square gradients of a neighborhood $D_{j}$ can be calculated by

$$
\left[\begin{array}{c}
\left.\bar{g}_{s x}\right|_{D_{j}} \\
\left.\bar{g}_{s y}\right|_{D_{j}}
\end{array}\right]=\left[\begin{array}{l}
\sum_{i \in D_{j}}\left(g_{s x}\right)_{i} \\
\sum_{i \in D_{j}}\left(g_{s y}\right)_{i}
\end{array}\right],
$$

where $i$ is at each pixel in the neighborhood. Since $D_{j}$ can be partitioned into four base blocks, (3) is also equal to the sum of the local averaged square gradients of base blocks. For instance, since $D_{1}=\{I, I I, I V, V\}$,

$$
\begin{aligned}
\left.\bar{g}_{s x}\right|_{D_{1}} & =\sum_{i \in I}\left(g_{s x}\right)_{i}+\sum_{i \in I I}\left(g_{s x}\right)_{i}+\sum_{i \in I V}\left(g_{s x}\right)_{i}+\sum_{i \in V}\left(g_{s x}\right)_{i} \\
& =\left.\bar{g}_{s x}\right|_{I}+\left.\bar{g}_{s x}\right|_{I I}+\left.\bar{g}_{s x}\right|_{I V}+\left.\bar{g}_{s x}\right|_{V} .
\end{aligned}
$$

In the same way, we have

$$
\left.\bar{g}_{s y}\right|_{D_{1}}=\left.\bar{g}_{s y}\right|_{I}+\left.\bar{g}_{s y}\right|_{I I}+\left.\bar{g}_{s y}\right|_{I V}+\left.\bar{g}_{s y}\right|_{V} .
$$

Because of the general parallel and anisotropy features, ridge patterns from the four overlapping neighborhoods of

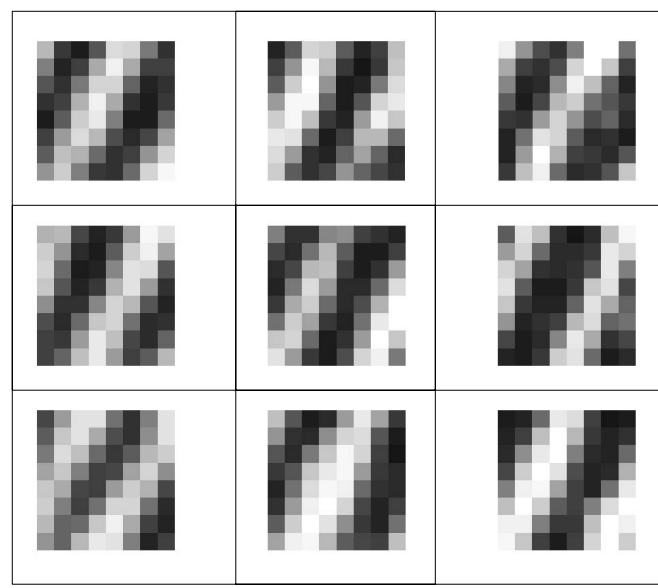

Figure 3. A real fingerprint example site of $3 \times 3$ image blocks: paralleled ridge pattern.

the target block are highly correlated to each other. If the window size is small, the ridge lines over the whole site are paralleled and slowly varied as shown in Figure 3. That is, the local orientation estimates from the four overlapping neighborhoods are almost the same, and they are very close to that of the target block. Therefore, the ridge patterns in the overlapping neighborhoods can be viewed as different images of similar figures. It is well known in digital image processing that redundant estimations are useful in recovering noisy images [2]. Analogously, if the target block is smeared by uncorrelated random noise, it is also possible to approximate the dominant orientation using information from its neighborhoods. The average of the averaged square gradients $\left[\left.\bar{g}_{s x}\right|_{D_{j}},\left.\bar{g}_{s y}\right|_{D_{j}}\right]^{T}$ is:

$$
\left[\begin{array}{l}
\overline{\bar{g}}_{s x} \\
\overline{\bar{g}}_{s y}
\end{array}\right]=\left[\begin{array}{l}
\left.\sum_{j=1 . .4} \bar{g}_{s x}\right|_{D_{j}} \\
\left.\sum_{j=1 . .4} \bar{g}_{s y}\right|_{D_{j}}
\end{array}\right] .
$$

Substituting the local averaged square gradients of the base blocks, such as (4) and (5), into (6) yields

$$
\left[\begin{array}{l}
\overline{\bar{g}}_{s x} \\
\overline{\bar{g}}_{s y}
\end{array}\right]=\left[\begin{array}{l}
\left.\sum_{\Gamma} r_{\Gamma} \cdot \bar{g}_{s x}\right|_{\Gamma} \\
\sum_{\Gamma} r_{\Gamma} \cdot \bar{g}_{s y} \mid \Gamma
\end{array}\right]
$$

where $\Gamma=I, I I, . ., I X$ represents any base block in the site. $r_{\Gamma}$ is a constant that counts the times of base block $\Gamma$ involving in the estimation, which is equal to the number of neighborhoods it belongs to. Note that $\left[\left.\bar{g}_{s x}\right|_{\Gamma},\left.\bar{g}_{s y}\right|_{\Gamma}\right]^{T}$ can be calculated using (1).

We can further normalize the constants $r_{\Gamma}$ in (7) so that $\sum \frac{r_{\Gamma}}{K}=1$, where $K=\sum r_{\Gamma}$. We denote $\sum \frac{r_{\Gamma}}{K}=1$ as the overlapping ratio. Using again the example in Figure 2, we see that the centered target block $V$ has the highest overlapping ratio of $\frac{4}{16}$, while its adjacent blocks $I I, I V, V I, V I I I$ 
have a ratio of $\frac{2}{16}$ and the rest at the corners have the least overlapping ratio of $\frac{1}{16}$. This means that the base blocks in a site are not equally important in the redundant estimation. The centered target block itself weights most, its edgetouched blocks come in second, followed by its cornertouched blocks.

In reality, the ridges are not always smooth and clearly separated, especially at the presence of noise. The dirt links parallel ridges together and results in poor separation. Since the orientations of uncorrelated noise can be arbitrarily distributed in all directions, the anisotropy feature of ridge structures is corrupted in the smudged areas. However, the base blocks are not uniformly contaminated depending on the noise position and size. Therefore, we need to modify (7) to construct a weighting factor that can compensate for different noise effects.

Recall in Section 2, the coherence is a reliability measure that indicates the anisotropic degree of ridge flows in an observation window. The coherence measure ranges from 0 to 1 . If the coherence value is equal to 0 , it means the gradients are equally distributed over all directions. On the contrary, if the coherence value is equal to 1 , it indicates all squared gradient vectors share the same orientation angle.

We compute the associated coherence $C_{\Gamma}$ for each base block $\Gamma$ using (2), and multiply it with the overlapping ratio to produce a weighting factor $w_{\Gamma}$ :

$$
w_{\Gamma}=C_{\Gamma} \cdot \frac{r_{\Gamma}}{K} .
$$

Note that in an ideal case where $C_{\Gamma}=1$ (meaning that all ridge lines in $\Gamma$ have the same orientation angle),

$$
\sum w_{\Gamma}=\sum C_{\Gamma} \cdot \frac{r_{\Gamma}}{K}=C_{\Gamma} \sum \frac{r_{\Gamma}}{K}=1 .
$$

If the assumption relaxes to the whole site, we will have

$$
\left[\begin{array}{l}
\left.\bar{g}_{s x}\right|_{\Gamma} \\
\left.\bar{g}_{s y}\right|_{\Gamma}
\end{array}\right]=\left[\begin{array}{l}
\left.\bar{g}_{s x}\right|_{V} \\
\left.\bar{g}_{s y}\right|_{V}
\end{array}\right] .
$$

According to (9) and (10),

$$
\left[\begin{array}{l}
\overline{\bar{g}}_{s x} \\
\overline{\bar{g}}_{s y}
\end{array}\right]=\left[\begin{array}{l}
\left.\sum w_{\Gamma} \cdot \bar{g}_{s x}\right|_{\Gamma} \\
\left.\sum w_{\Gamma} \cdot \bar{g}_{s y}\right|_{\Gamma}
\end{array}\right]=\left[\begin{array}{l}
\left.\bar{g}_{s x}\right|_{V} \cdot \sum w_{\Gamma} \\
\left.\bar{g}_{s y}\right|_{V} \cdot \sum w_{\Gamma}
\end{array}\right]=\left[\begin{array}{l}
\left.\bar{g}_{s x}\right|_{V} \\
\left.\bar{g}_{s y}\right|_{V}
\end{array}\right] .
$$

While in normal cases where $C_{\Gamma} \neq 1$, replacing $r_{\Gamma}$ in (7) with $w_{\Gamma}$ in (8) yields:

$$
\left[\begin{array}{l}
\overline{\bar{g}}_{s x} \\
\overline{\bar{g}}_{s y}
\end{array}\right]=\left[\begin{array}{l}
\left.\sum w_{\Gamma} \cdot \bar{g}_{s x}\right|_{\Gamma} \\
\left.\sum w_{\Gamma} \cdot \bar{g}_{s y}\right|_{\Gamma}
\end{array}\right]=\left[\begin{array}{l}
\left.\sum C_{\Gamma} \frac{r_{\Gamma}}{K} \cdot \bar{g}_{s x}\right|_{\Gamma} \\
\left.\sum C_{\Gamma} \frac{r_{\Gamma}}{K} \cdot \bar{g}_{s y}\right|_{\Gamma}
\end{array}\right] .
$$

As introduced in Section 2, the orientation angle is orthogonal to the gradient angle at the edge of a ridge line. Therefore, the dominant orientation angle $\theta_{V}$ of the target block $V$ can be estimated from the average of the averaged squared gradients in (11) by:

$$
\theta_{V}=\frac{1}{2} \angle\left(\overline{\bar{g}}_{s x}, \overline{\bar{g}}_{s y}\right)+\frac{\pi}{2} \text {. }
$$

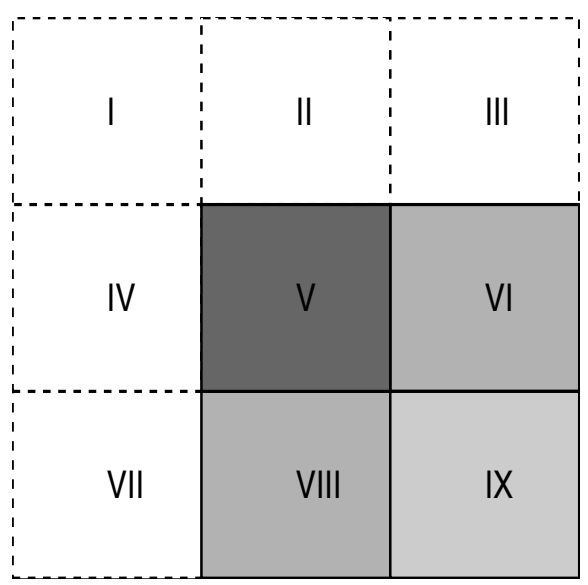

\section{Figure 4. A patched site where the target block is at an edge.}

Note that $\angle\left(\overline{\bar{g}}_{s x}, \overline{\bar{g}}_{s y}\right)$ is within $[-\pi, \pi)$ because of the doubled gradient angle and the use of arctangent function $\operatorname{atan}(\cdot)$, while $\theta_{V}$ is within $[0, \pi)$ as being desired.

For a target block at an edge, we can apply the same algorithm by patching the site with virtual blocks of which the gradients are assumed to be zeros. An example of such scheme is illustrated in Figure 4.

From an algorithmic point of view, the above algorithm simply takes the weighted average on a base block level. Unlike in some literatures, where the coherence measure is used directly as the weighting factor in estimation [14], we incorporate the anisotropic measure with an overlapping ratio that also indicates the weight of relative position. The concept of overlapping ratio initially comes from the idea of conducting redundant estimation of orientation fields over four overlapping neighborhoods.

In the next section, we will present experiment results to show the performance of our weighted averaging algorithm in comparison with other gradient based methods.

\section{Experimental Results}

We test our weighted averaging method for estimation of orientation fields on real fingerprint images from FVC2000's sample databases [8]. All the tested fingerprint images are suffering from different noise effects caused by large creases, callus, moist or smudges. Due to limited space, six different fingerprint images from five major fingerprint categories [8] (arch, tented arch, loop, twin loop and whorl) are presented in this session. For the purpose of comparison, three gradient based algorithms are evaluated on these fingerprint images: the conventional method $[1,11]$, the hierarchical method [4] and our weighted averaging method. 


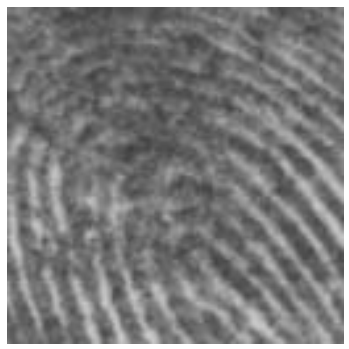

(a)

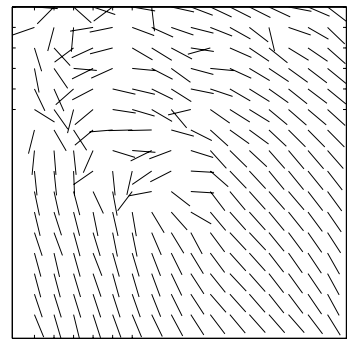

(c)

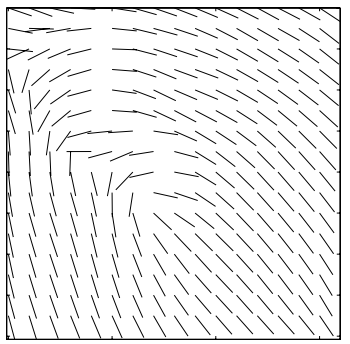

(d)

Figure 5. (a) A loop type fingerprint segment with smudged areas on top left; (b) the coherence map; (c) orientation estimates produced by the conventional method; (d) orientation estimates produced by our weighted averaging method.

Since no ground truth exists for the orientation field of fingerprints, it is difficult to construct a general measure to evaluate the objective error quantitatively $[12,16]$. Hence, the quality of an estimation has to be assessed by means of manual inspection. In the following, we will display the estimation results to demonstrate that the performance of our algorithm is superior to the other two gradient based methods with respect to noise resistance.

Figure 5(a) is a smudged segment cropped from a fingerprint image. The ridge pattern contains an indistinct left loop structure because the upper left corner is smeared by noise. To extract the orientation fields, we first divide the segment into small grids. The associated coherence level of each grid is printed in Figure 5(b). We see the smudged areas (upper left) are brighter in Figure 5(b), which means the coherence values at those positions are lower. Figure 5(c) is the estimation results from the conventional method, where the orientation fields are in a clutter at the top left. Figure 5(d) shows a set of estimates using our weighted averaging method. One can see that the left loop structure is clearly

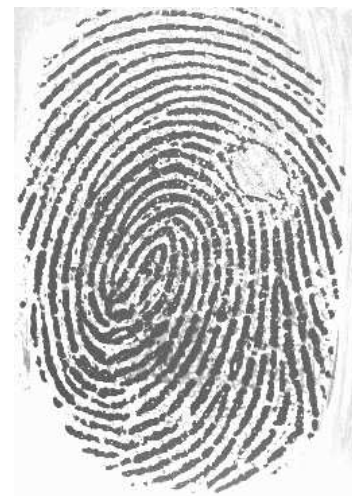

(a)

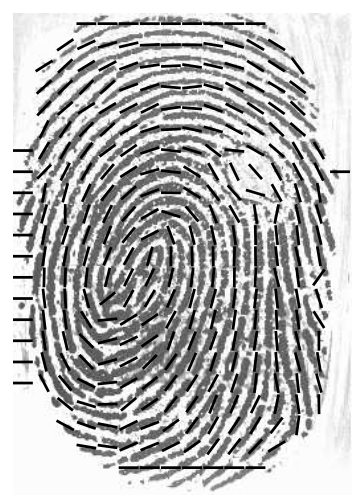

(c)

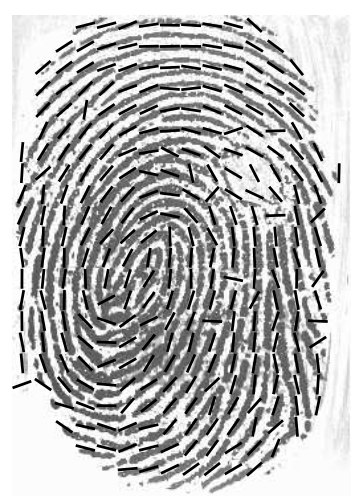

(b)

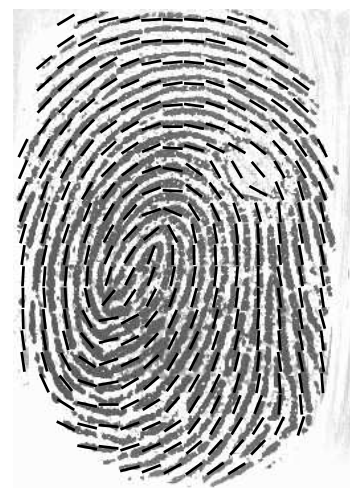

(d)
Figure 6. (a) A twin loop fingerprint image with a bubble blotch on top right; orientation fields estimated from: (b) the conventional gradient based algorithm; (c) the hierarchical scheme; (d) our weighted averaging method.

revealed in Figure 5(d).

Figure 6 is a set of estimation results for a complete twin loop fingerprint. A big bubble blotch, possibly caused by callus, appears at the upper right hand site of the original image as shown in Figure 6(a). We then superimpose the estimated orientation fields from different gradient based methods on the original fingerprint image and display the results in Figure 6(b), 6(c) and 6(d) respectively. Figure 6(b) is produced from the conventional method, where several spots are disturbed by noise. Although the estimation is improved by using the hierarchical scheme, the orientation fields are not yet smooth as shown in Figure 6(c). Among the three, our weighted averaging method performs the best by recovering the ridge structures and producing smoother 


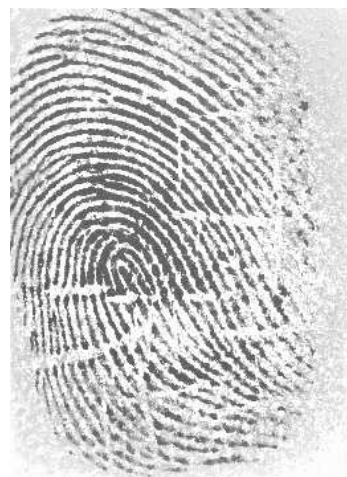

(a)

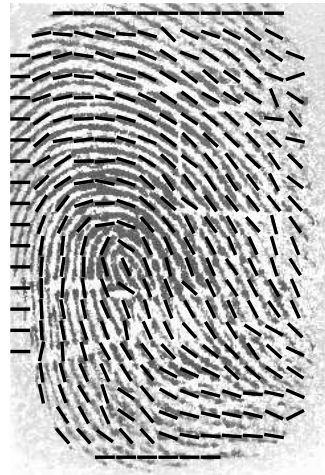

(c)

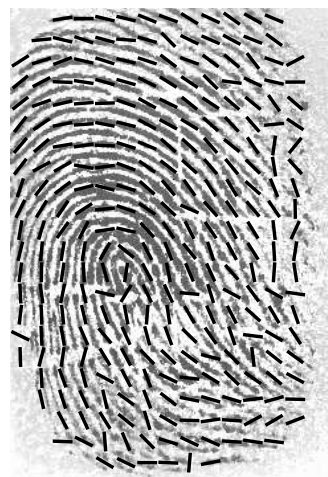

(b)

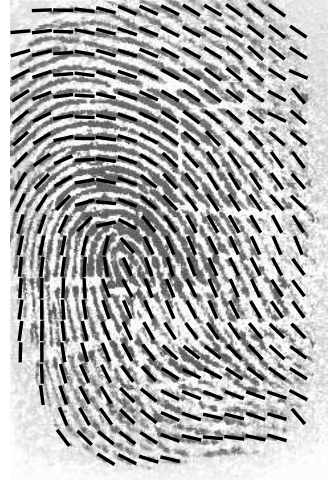

(d)

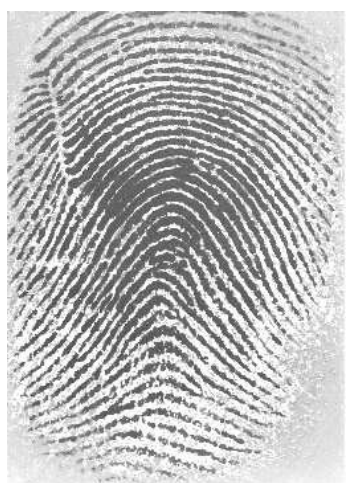

(a)

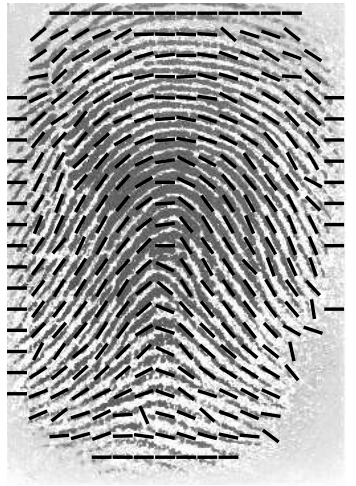

(c)

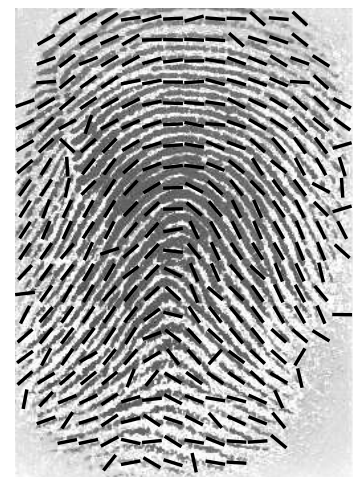

(b)

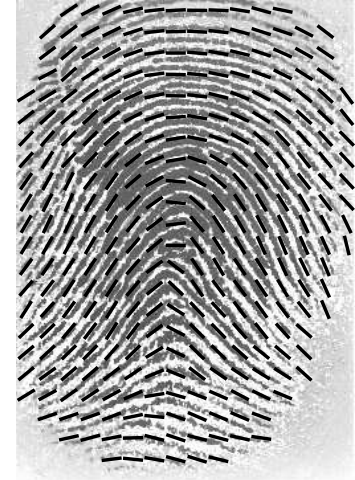

(d)
Figure 7. (a) A loop type fingerprint image with several vertical and horizontal creases; orientation fields estimated from: (b) the conventional method; (c) the Hierarchical scheme; (d) our enhanced algorithm.

estimates around the blotch. This is demonstrated in Figure 6(d).

Figure 7(a) is another left loop type fingerprint but with a complete image. We see in the figure there are several vertical and horizontal creases of different lengths run through the fingerprint. From figure 7(b), 7(c) and 7(d), we can also conclude that the weighted averaging method again achieves better estimation results for the orientation fields than the other gradient based methods.

Figure 8, Figure 9 and Figure 10 are experiment results for arch, tented arch and whorl type fingerprints respectively. In Figure 8, there is a scar run through top left of the fingerprint. While the fingerprints shown in Figure 9 and Figure 10 are affected by different level of moist and creases. For these fingerprint examples, the results show that our weighted averaging method is capable to extract the information of ridge direction reliably and it is more robust against noise in comparison with the other two gradient based methods.

In general from our experiments, we find that the enhanced gradient based method is able to yield satisfactory estimation for most types of fingerprints. However, it lacks of strength to extract high curvatures such as at the core of a whorl type fingerprint. This is mainly because of the nature of averaging process when the dominant orientation is calculated. A possible solution to this problem is using post processing techniques such as mathematical modeling, which is beyond the scope of this paper. 


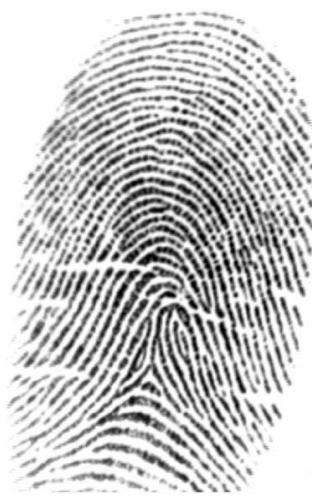

(a)

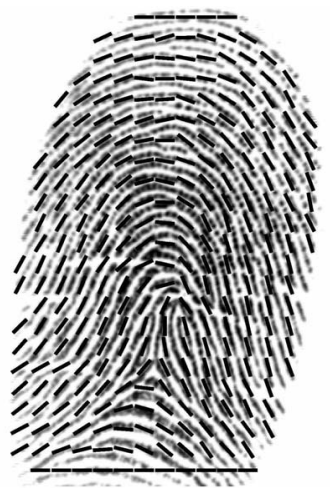

(c)

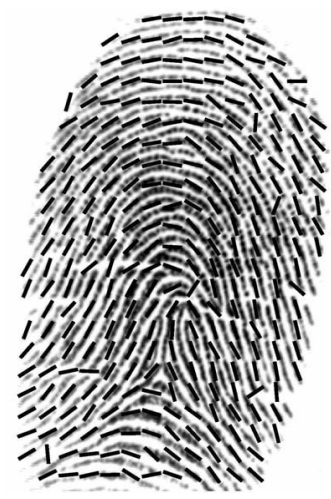

(b)

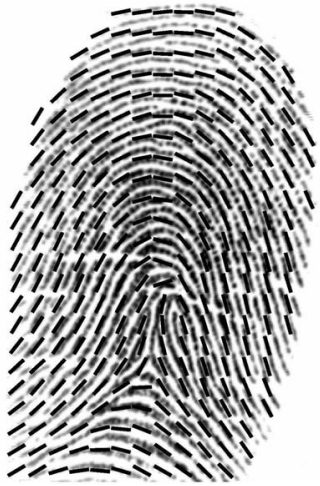

(d)

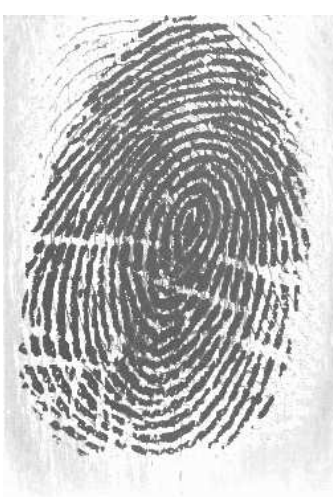

(a)

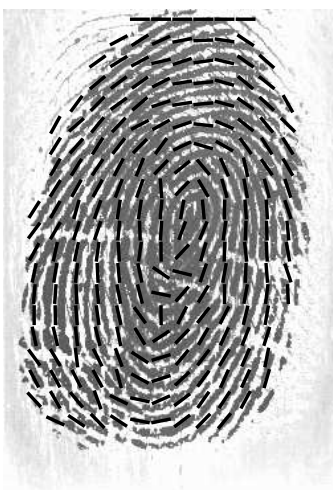

(c)

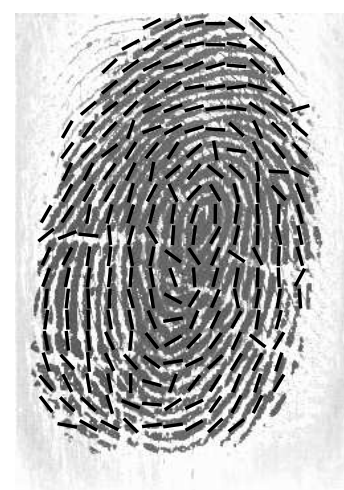

(b)

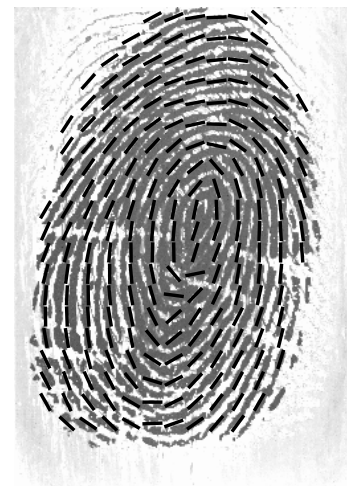

(d)
Figure 9. (a) A tented arch type fingerprint image with horizontal creases; orientation fields estimated from: (b) the conventional method; (c) the Hierarchical scheme; (d) our enhanced algorithm.

\section{Conclusions}

In this paper, we have proposed a new gradient based algorithm that is more robust against noise for estimation of fingerprint orientation fields. Our initial idea is to conduct redundant estimation on overlapping neighborhoods around a target block, which can exploit the parallel and anisotropic features of fingerprint ridge patterns. Following this idea, we propose an approach that averages a series of weighted estimates for every base block of which the dominant orientation is to be estimated. The weighting factor consists of a coherence measure that indicates the anisotropic level of a base block, and a predefined overlapping ratio evaluated from the idea of redundant estimation. For the purpose of comparison as well as evaluation, we test the proposed algorithm together with other popular gradient based methods on real fingerprint images from a standard database. The fingerprint images are selected from different categories and all suffering from obvious noise effects. Our preliminary experiment results show that the proposed weighted averaging method is superior with respect to noise resistance.

\section{Acknowledgement}

The work is financially supported by the ARC linkage project LP0455324. 


\section{References}

[1] A. M. Bazen and S. H. Gerez. Systematic methods for the computation of the directional fields and singular points of fingerprints. IEEE Trans. Pattern Anal. Machine Intell., 24(7):905-919, Jul 2002.

[2] R. C. Gonzalez and R. E. Woods. Digital Image Processing. Prentice Hall, 2001.

[3] A. Jain, S. Prabhakar, L. Hong, and S. Pankanti. Filterbankbased fingerprint matching. In IEEE Trans. Image Processing, volume 9, pages 846-859, May 2000.

[4] A. K. Jain, L. Hong, and R. Bolle. On-line fingerprint verification. IEEE Trans. Pattern Anal. Machine Intell., 19(4):302-314, Apr 1997.

[5] A. K. Jain, S. Pankanti, and L. Hong. A multichannel approach to fingerprint classification. IEEE Trans. Pattern Anal. Machine Intell., 21(4):348-359, Apr 1999.

[6] M. Kass and A. Witkin. Analyzing oriented patterns. Computer Vision, Graph and Image Processing, 37(3):362-385, Mar 1987.

[7] M. Kawagoe and A. Tojo. Fingerprint pattern classification. Pattern Recognition, 17(3):295-303, 1984.

[8] D. Maltoni, D. Maio, A. K. Jain, and S. Prabhakar. Handbook of Fingerprint Recognition. Springer-Verlag, New York, 2003. RMIT Swanston: 363.258 H236.

[9] B. M. Mehtre. Fingerprint image analysis for automatic identification. Machine Vision and Applications, 6:124-189, 1993.

[10] S. Pankanti, S. Prabhakar, and A. K. Jain. On the individuality of fingerprints. In IEEE Trans. Pattern Anal. Machine Intell., volume 24, pages 1010-1025, Aug 2002.

[11] A. R. Rao and R. C. Jain. Computerized flow field analysis: oriented texture fields. IEEE Trans. Pattern Anal. Machine Intell., 14(7):693-709, Jul 1992.

[12] N. Ratha, S. Chen, and A. K. Jain. Adaptive flow orientation based feature extraction in fingerprint images. Pattern Recognition, 28:1657-1672, Nov 1995

[13] B. G. Sherlock, D. M. Monro, and K. Millard. Fingerprint enhancement by directional fourier filtering. In IEE Proc. Vision, Image and Signal Processing, volume 141, pages 87-94, Apr 1994.

[14] C. F. Shu and R. C. Jain. Vector field analysis for oriented patterns. IEEE Trans. Pattern Anal. Machine Intell., 40(3), 2001.

[15] C. L. Wilson, G. T. Candela, and C. I. Watson. Neural network fingerprint classification. Journal of Artificial Neural Networks, 1(2):203-228, 1994.

[16] J. Zhou and J. Gu. A model-based method for the computation of fingerprints' orientation field. IEEE Trans. Image Processing, 13(6):821-835, 2004. 